\title{
EJECTION OF MATTER AND ENERGY FROM NGC 4258
}

\author{
E.M. Burbidge and G. Burbidge \\ Department of Physics and Center for Astrophysics \& Space Sciences \\ University of California, San Diego \\ La Jolla, California 92093-0111, USA
}

\begin{abstract}
It has been claimed that the megamaser observations of the nucleus of NGC 4258 show that a massive black hole is present in its center (Miyoshi et al. 1995, Greenhill et al. 1995). We show that the evidence of ejection of gas, radio plasma, and X-ray emitting QSOs from this nucleus all show that the ejection is coming from the center in a curving flow within a cone with angle $\sim 40^{\circ}$, centered at P.A. $100^{\circ}$. This is close to the direction in which the velocities from the megamaser have been measured, so that the evidence taken as a whole suggests that the masering gas also is being ejected in the same direction at velocities $\pm 900 \mathrm{~km} \mathrm{sec}^{-1}$ and not rotating about a massive black hole. Thus it does not provide evidence for a black hole in the center.
\end{abstract}

Subject headings: galaxies: nuclei: individual (NGC 4258) - black holes - masers

\section{INTRODUCTION}

There are no new observations in this paper. Its purpose is to summarize evidence collected since 1962 from the measurements of ionized gas, continuum radio observations, and X-rays, all of which shows that violent activity taking place close to the center of NGC 4258 is giving rise to ejection of gas, relativistic particles and coherent objects and to show that these observations together with the megamaser observations all suggest that matter is being ejected from the center. However, contrary to what has been frequently claimed from the megamaser observations alone (Miyoshi et al. 1995), they do not provide evidence for the existence of a massive black hole.

\section{OPTICAL, RADIO AND X-RAY OBSERVATIONS OF NGC 4258}

NGC 4258 is one of the nearest galaxies with an active nucleus. Its distance $D \approx 7 M p c$, although some investigators have used a smaller value. Its systemic velocity $V_{\circ}=465 \mathrm{~km}$ $\mathrm{s}^{-1}$. The low-ionization strong emission lines in the nucleus lead to a classification as a LINER galaxy rather than as a Seyfert galaxy, although it was in Seyfert's original list (cf Burbidge \& Burbidge 1962). 
A study of the velocity field in the ionized gas in the bright knotty spiral arms was made as part of our original program of measurement of rotation curves of spiral galaxies (Burbidge, Burbidge \& Prendergast 1963). This showed the galaxy is quite massive $\left(\sim 10^{11} M_{\odot}\right.$ interior to our last measured point), but it also showed that substantial non-circular motions were present within 40 arcsec of the apparent nucleus. In that study, a position angle of $157^{\circ}$ was taken to be the direction of the major axis. The "non-circular" motion occurred in P.A. $157^{\circ}, 167-169^{\circ}$, and $126^{\circ}$ (see Fig 1 for these directions). A formal analysis of these velocities in terms of the mass model of similar spheroidal surfaces, balancing rotation against the gravitational pull of the mass interior to the measured points, gave, for any choice of axial ratio, the unphysical result of negative densities at $\sim 1$ arc min from the center. Burbidge et al. hence concluded that radial motions of $\sim 300 \mathrm{~km} \mathrm{~s}^{-1}$ were present, and suggested that the mass distribution of NGC 4258 might resemble a barred spiral.

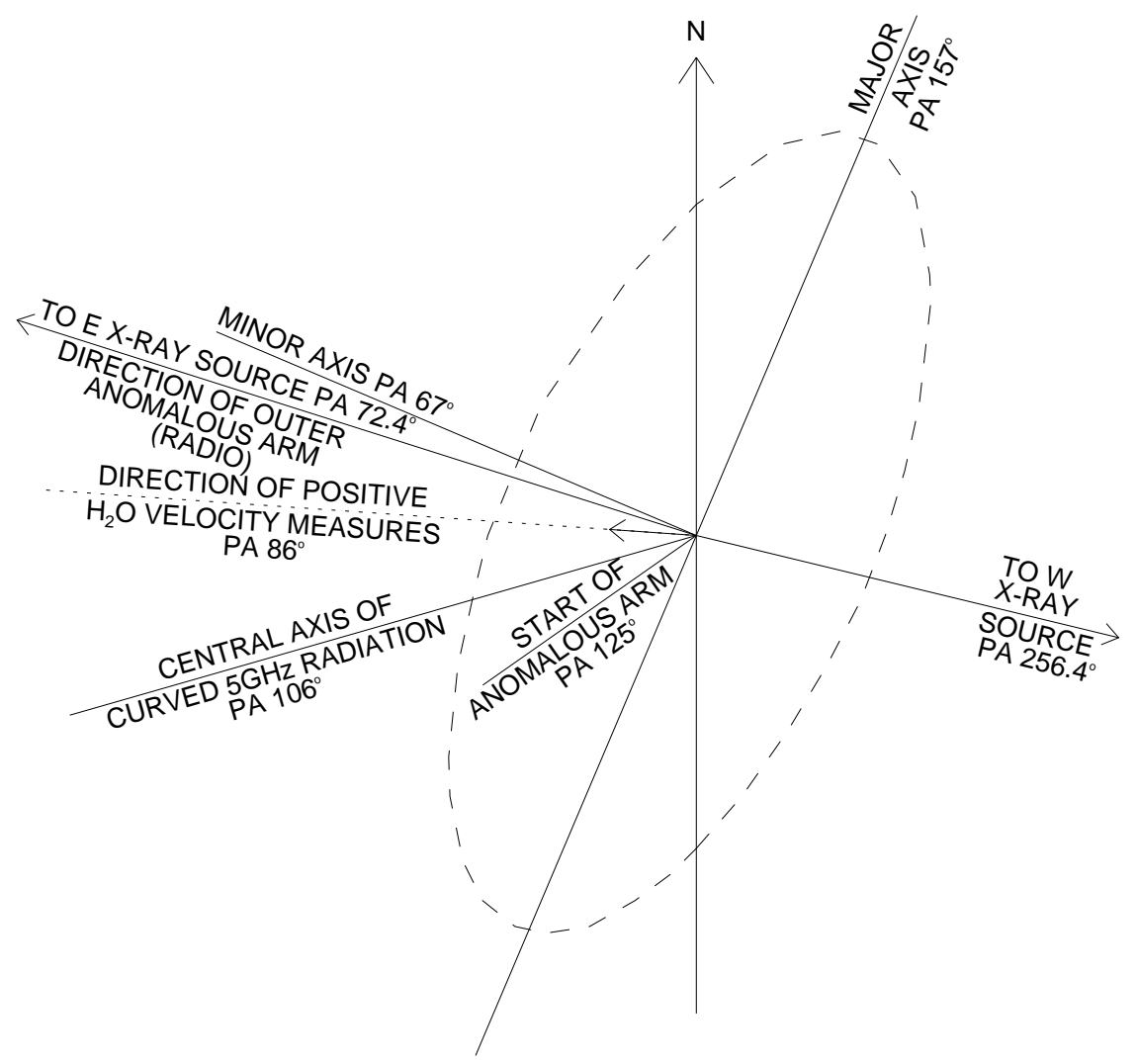

Figure 1: Schematic diagram of NGC 4258 showing position angles of major and minor axes, and various directions in the plane of the sky of apparent ejection from the center (ionized gas, relativistic particles for radio emission, X-ray sources).

The existence of non-circular motions in NGC 4258 was confirmed by several later studies (cf. van der Kruit 1974), and they were linked to the discovery by Courtès and Cruvellier (1961) of a pair of "anomalous arms" in P.A. 125 . Optically, these are visible in $\mathrm{H} \alpha$ emission only. Since these arms have no stellar continuum from stars expected to be present to ionize the hydrogen (Courtès et al. 1993), it was concluded that these "arms" 
are not normal quasi-stationary density concentrations, but hot gas which is being ejected.

Van der Kruit, Oort \& Mathewson (1972) measured the radio continuum at $1415 \mathrm{MHz}$, and found smooth curved ridges of continuum which differ in position, shape, and continuity from the normal optical arms, but close to the center they coincide with the filamentary $\mathrm{H} \alpha$ arms of Courtès \& Cruvellier. This study of the optical and radio data led van der Kruit et al. to the conclusion that there had been ejection from the nucleus of clouds in two opposite directions about $18 \times 10^{6}$ years ago, at velocities ranging from 800 to $1600 \mathrm{~km} \mathrm{~s}^{-1}$.

High resolution radio continuum observations made at $1480 \mathrm{MHz}$ by van Albada \& van der Hulst (1982) showed that the radio emission that lies along the SE anomalous arm curves around sharply eastward and ends in a tail directed toward the NE. Even more striking detail is shown in Fig 2c of the paper by Cecil et al. (1995), which is based on measurements at $5 \mathrm{GHz}$. The position angle of the central axis of this emission near the center, $106^{\circ}$, is shown in Fig 1.

Cecil et al. (1995) also used data from Ford et al. (1986), who discovered that the Courtès \& Cruvellier anomalous arms or jets were resolved into distinct helical strands braided around each other, suggesting the influence of a magnetic field in the center reacting with the outward flow of the plasma. A combination of X-ray data with the optical and radio data led Cecil et al. to a model in which hot shocked gas at $500 \mathrm{~km} \mathrm{~s}^{-1}$ might have been entrained as the outward-moving nuclear jets reacted with the molecular clouds of $\mathrm{CO}$ that had been mapped as lying closely along the anomalous arms (Krause et al. 1990). The strength of a magnetic field along the anomalous arms is indicated by the high degree of polarization in the radio emission at $1.49 \mathrm{GHz}$ measured by Hummel et al. (1989). Their Fig 3 shows this very clearly, and they point out that the magnetic field configuration is bisymmetric, with the field lines closely following the ridges of the radio emission.

A detailed study of the emission-line velocity field in the central few arc min, by Rubin \& Graham (1990) using the KPNO echelle spectrograph on the 4-m Mayall telescope, shows the complexity of the ionized gas flow close to the nucleus. Their plots of velocity in PA $150^{\circ}$ and $125^{\circ}$ reveal multiple-valued velocities, and they described the velocity details as "more spectacular than those we have seen in any other galaxy". Rubin \& Graham suggested that this indicated that these are due to jets bursting out of the galactic plane, with gas drizzling back into the plane at positions indicated on their velocity plots.

All of these studies are best explained by high velocity jets emanating from the nucleus, including (or being initiated by) relativistic electron (or electron-positron) jets emitting synchrotron radiation, as indicated by the radio data.

ROSAT PSPC X-ray observations of NGC 4258 and its vicinity (Pietsch et al. 1994) showed the anomalous arms or jets in X-rays for the first time, most clearly in the hard (H2) band where absorption due to the disk of the galaxy is minimum. These data also showed two bright compact X-ray sources, each $\sim 9$ arc min from the center of the galaxy, the $\mathrm{E}$ source in P.A. $72 .^{\circ} 4$ with respect to the center and the $\mathrm{W}$ source in P.A. $256^{\circ}$ 4; these 
directions are shown in Fig 1. The line joining the two sources passes close to the center and lies within some $5^{\circ}$ of the minor axis. An overlay of the directions to compact external sources on the $1480 \mathrm{MHz}$ radio map of van Albada \& van der Hulst (Fig 8 of Pietsch et al.) shows this quite clearly, and Pietsch et al. point out that the line connecting the compact sources touches the ends of the anomalous arms. Following this suggestion by Pietsch et al. that the X-ray compact sources might have been ejected from the nucleus of NGC 4258, Burbidge (1995) obtained spectra of them and showed that they are indeed a pair of QSOs with redshifts $z=0.398$ (W source) and 0.653 (E source), and discussed the implications if these have been ejected from the nucleus of NGC 4258.

\section{MEGAMASER OBSERVATIONS AND THEIR INTERPRETATIONS}

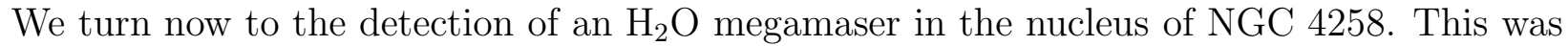
reported by Claussen et al. (1984), and Miyoshi et al. (1995) detected maser emission at velocities offset by \pm 750 to $1000 \mathrm{~km} \mathrm{~s}^{-1}$, bracketing the emission at the systemic velocity of $\pm 465 \mathrm{~km} \mathrm{~s}^{-1}$. They have concluded that relative to the center the velocities of the excited gas cover the range $\pm 900 \mathrm{~km} \mathrm{~s}^{-1}$. The figure of their model shows blobs spaced almost equidistantly on the high-velocity side. According to their model, the velocities and spacing relative to the nucleus of NGC 4258 are perpendicular to the line of sight, and arise in a disk oriented in P.A. $86^{\circ}$, at an angle $\sim 11^{\circ}$ to the minor axis of NGC 4258 as estimated from its image (Cecil et al. 1995) (see Fig 1). They quoted Hubble (1943) who had determined from the dark lanes that the spiral arms are trailing. Miyoshi et al. give their determination of the angle between the spin axis of this $\mathrm{H}_{2} \mathrm{O}$ megamaser disk and the spin axis of the galaxy as $119^{\circ}$. Miyoshi et al. chose to interpret their velocity observations as being due to circular motion involving a disk surrounding a massive black hole. Thus they have claimed that their observations provide direct evidence for the existence of a massive black hole in the center of NGC 4258.

Now it is well known that velocity shifts alone detected in the central region of a galaxy may be due to rotation, or to radial motion inward or outward, or to a combination of rotation and radial motion. Since one is trying to construct a 3-dimensional model from 2-dimensional data, without an independent measurement of the position angle of the line of nodes, or velocity measurements in many different position angles, it is impossible to determine whether the motions are purely circular or radial or both. However, what has been done by Miyoshi et al. is to assume without proof that they are looking at circular motion about a black hole - i.e. they assume that the black hole-accretion disk paradigm is correct, and in so doing determine the position angle of the rotating disk as $86^{\circ}$. In this way they are able to calculate a central mass, and this is being widely claimed to be observational evidence for the presence of a massive black hole. For other galaxies with megamasers in which no motions have been measured, reference is made to NGC 4258 as the evidence for a black hole. Thus the idea is growing up that the existence of megamasers is a further indication of the existence of massive black holes in active galaxies. This is a good example of the disservice that belief in the paradigm is doing to a true understanding of the activity in galaxies. 
However, the fact is that the direction of the velocity vectors directly observed from the maser line components is close to that for optical, radio and X-ray ejections (Arp 1996). This is shown in Fig 1. Thus the simplest explanation devoid of any further assumption, is that the gas excited in the masering process is being ejected by the same unknown process and in the same direction as all of the matter and energy further out. This may be connected with the linear drift of $9.5 \mathrm{~km} \mathrm{~s}^{-1} \mathrm{yr}^{-1}$ in those features with velocities $\pm 150 \mathrm{~km}$ $\mathrm{s}^{-1}$ of the systemic velocity, while the overall velocity range remains stationary over time (Greenhill et al. 1995a).

\section{CONCLUSION}

As we have shown all of the evidence from optical, radio continuum and X-ray studies suggests that explosive events in the nucleus have given rise to this activity. Whatever the process is, it has restricted the ejection to a cone of angle $\sim 40^{\circ}$ containing the minor axis of NGC 4258, and approximately centered on the minor axis. The megamaser observations fit very well into this picture, since the P.A. of the velocities, $86^{\circ}$, is only $\sim 11^{\circ}$ from the direction of the minor axis.

Thus the observations taken as a whole point towards ejection in the same cone, not rotation about a black hole. By arguing that the megamaser observations are to be interpreted as evidence of rotation, Myoshi et al. have to arrange that the rotation axis of the purported disk is oriented practically at right angles to the direction of ejection of the other material.

No one knows what the primary source of energy is. It may be gravitational energy released in the collective evolution of a dense star cluster, or a massive black hole, or the creation of matter at the nucleus. While the most popular model is based on the release of gravitational energy in the black hole-accretion disk model (cf Rees 1984), and this has turned into the black hole-accretion disk paradigm, the observations do not necessarily indicate that this is correct. Unfortunately the general tendency of observers has been to interpret the observations in terms of this model rather than testing it (cf Holt et al. 1992). The simplest model required to explain all of the observations of all of the activity in the nucleus of NGC 4258 must be able

1. To provide energy to excite and maintain what appear to be a number of regularly spaced gas clouds as is indicated by the maser observations.

2. To provide energy to eject gas at high speed $\left(\sim 1000 \mathrm{~km} \mathrm{~s}^{-1}\right)$ over time scales of $\sim 10^{6}-10^{7}$ years or longer.

3. To provide energy that will generate radio synchrotron emission giving rise to the complex radio arms.

4. To eject compact X-ray sources each centered on QSOs with anomalous redshifts. 
There is certainly a large mass concentration in the central region of NGC 4258, but evidence for a black hole is only obtained by interpreting the megamaser observations in a special way, and ignoring the other evidence. Optical spectroscopy with high spatial resolution in the central 10-20 arc sec may help to define the properties of the nuclear region.

This research was supported in part by NASA grant NAG5-1630. 


\section{REFERENCES}

Arp, H.C. 1996, private communication

Burbidge, E.M. 1995, A\&A, 298, L1

Burbidge, E.M. \& Burbidge, G.R. 1962, ApJ, 135, 694

Burbidge, E.M., Burbidge, G.R. \& Prendergast, K.H. 1963, ApJ, 138, 375

Cecil, G., Wilson, A.S. \& dePree, C. 1995, ApJ, 440, 181

Claussen, M., Heiligman, G., Lo, K.Y. 1984, Nature, 310, 298.

Courtès, G. \& Cruvellier, P. 1961, C. R. Acad. Sci. Paris, 253, 218

Courtès, G., Petit, H., Hua, C.T., Martin, P., Blecha, A., Huguenin, D. \& Golay, M. 1993, A\&A, 268, 419

Ford, H., Dahari, O., Jacoby, G.H., Crane, P.C. \& Ciardullo, R. 1986, ApJ, 311, L7

Greenhill, L.J., Jiang, D.R., Moran, J., Reid, M., Lo, K.Y. \& Claussen, M.J. 1995, ApJ, 440, L19

Greenhill, L.J., Henkel, C., Becker, R., Wilson, T.L. \& Wouterloot, J.G.A. 1995a, A\&A, 304,21

Holt, S.S., Neff, S.G. \& Urry, C.M. 1992 "Testing the AGM Paradigm", AIP Conf. Proc. 254

Hubble, E. 1943, ApJ, 97, 112

Hummel, E., Krause, M. \& Lesch, H. 1989, A\&A, 211, 266

Krause, M., Cox, P., Garcia-Barreto, J.A., Downes, D. 1990, A\&A, 233, L1

Miyoshi, M., Moran, J., Herrstein, J., Greenhill, L., Nakal, N., Diamond, P., Inone, M. 1995, Nature, 373, 127

Nakai, N., Inone, M. \& Miyoshi, M. 1993, Nature, 361, 45

Pietsch, W., Vogler, A., Kahabka, P., Jain, A., \& Klein, V. 1994, A\&A, 284, 386

Rees, M.J. 1984, ARA\&A, 32, 471

Rubin, V. \& Graham, J. 1990, ApJ, 362, L5

van Albada, G.D. \& van der Hulst, J.M., 1982, A\&A, 115, 263

van der Kruit, P.C. 1974, ApJ, 192, 1

van der Kruit, P.C., Oort, J.H. \& Mathewson, D.S. 1972, A\&A, 21, 169 